\title{
Vibrational Suspension of Light Sphere in a Tilted Rotating Cylinder with Liquid
}

\author{
Victor G. Kozlov and Stanislav V. Subbotin \\ Laboratory of Vibrational Hydromechanics, Perm State Humanitarian Pedagogical University, \\ Sibirskaya Street 24, Perm 614990, Russia \\ Correspondence should be addressed to Victor G. Kozlov; victorkozlov2012@mail.ru
}

Received 30 April 2013; Revised 4 January 2014; Accepted 6 February 2014; Published 16 March 2014

Academic Editor: Benjamin Soenarko

Copyright ( 2014 V. G. Kozlov and S. V. Subbotin. This is an open access article distributed under the Creative Commons Attribution License, which permits unrestricted use, distribution, and reproduction in any medium, provided the original work is properly cited.

\begin{abstract}
The dynamics of a light sphere in a quickly rotating inclined cylinder filled with liquid under transversal vibrations is experimentally investigated. Due to inertial oscillations of the sphere relative to the cavity, its rotation velocity differs from the cavity one. The intensification of the lagging motion of a sphere and the excitation of the outstripping differential rotation are possible under vibrations. It occurs in the resonant areas where the frequency of vibrations coincides with the fundamental frequency of the system. The position of the sphere in the center of the cylinder could be unstable. Different velocities of the sphere are matched with its various quasistationary positions on the axis of rotating cavity. In tilted rotating cylinder, the axial component of the gravity force appears; however, the light sphere does not float to the upper end wall but gets the stable position at a definite distance from it. It makes possible to provide a vibrational suspension of the light sphere in filled with liquid cavity rotating around the vertical axis. It is found that in the wide range of the cavity inclination angles the sphere position is determined by the dimensionless velocity of body differential rotation.
\end{abstract}

\section{Introduction}

Rotating hydrodynamic systems attract a great interest because of their wide distribution in nature. The density inhomogeneity of such systems ensures the nontrivial inertial properties and, therefore, possibility of controlling them using the vibrations [1]. For instance the action of transverse vibrations on the free boundary of the centrifuged liquid [2] leads to the excitation of an azimuthal wave and excitation of intensive averaged liquid flow. Similar resonance effects occur when the free flowing medium [3] or a light cylindrical body [4] are in rotating system instead of a gas phase. In the last case, the vibration leads to the oscillation of the body and the emergence of its intensive differential rotation relative to the cavity, called "vibrational hydrodynamic top." Description of the differential rotation in the two-dimensional formulation is given in [4]. The presence of a rotating force field in a rotating frame leads to the circular oscillations of the body. The arising inertial azimuthal wave in the fluid causes the pulsating motion in the viscous boundary layer. This leads to the excitation of an averaged torque, spinning up the body. The direction of body rotation (outstripping or lagging) is determined by the direction of the azimuthal wave. The existence of the lagging and outstripping rotation is natural for all centrifuged systems inhomogeneous in density. The resonance areas are defined by the ratio of vibration frequency to the rotation speed $n \equiv \Omega_{\text {vib }} / \Omega_{\text {rot }}$. The outstripping rotation of the body with respect to the cavity is excited if $n>1$ and the lagging one if $n<1$. The static external force field also leads to the oscillations of the light body relative to the cavity and its lagging differential rotation.

If the top has a spherical shape [5], the vibrations result in excitation of its differential rotation and positioning on the rotational axis. The positioning of bodies is an actual problem, particularly under the microgravity conditions. The phenomenon of "acoustic levitation" of liquid droplets in the gravity field $[6,7]$ could be an example. The levitation is carried out by the acoustic pressure in the standing wave.

The rotation of the cavity qualitatively changes the law of the body motion. In the rotating fluid, the emersion speed of 
a light body is much smaller than in a nonrotating one $[8,9]$. The additional drag force due to the formation of so-called Taylor-Proudman column appears [10].

The purpose of this work is to continue the research of the "vibrational hydrodynamic top", especially the effect of the sphere positioning at some distance from the end walls of the rotating horizontal cylinder [5]. The essence of the effect consists in the next, with increase of the speed of differential rotation that occurs under vibrations; the symmetrical sphere position relative to the cavity ends becomes unstable. The sphere shifts towards one of the cavity ends and occupies a stable position at some distance from it. This indicates the existence of the lift force acting on the body and directed along the axis which is associated with the hydrodynamic interaction of the body with the cavity end walls. To measure this force, the system could be tilted relative to the horizon. In inclined position, the lift force directed along the axis is balanced by the axial component of the body weight. We found that under intensive differential rotation the body could remain in suspended state even in the cavity rotating around the vertical axis.

\section{Experimental Setup and Techniques}

In a cylindrical cavity of circular cross section 1 (Figure 1), the light spherical body 2 is placed. The cavity filled with a liquid is set in a horizontal position on table 3 of an electrodynamic vibrator, providing the translational vibrations perpendicular to the rotation axis. The length and the radius of the cavity are $L=7.20 \mathrm{~cm}$ and $R=2.60 \mathrm{~cm}$, respectively, the radius of the sphere is $r=1.77 \mathrm{~cm}$; the average density $\rho_{s}=0.17 \mathrm{~g} / \mathrm{cm}^{3}$. The relative size of the sphere is $2 r / L=0.49$. The working fluid is water.

A stepper motor 4 leads the cavity to the uniform rotation with frequency $f_{\text {rot }}=\Omega_{\text {rot }} / 2 \pi$. The instability of the cavity rotation is less than $0.001 \mathrm{rps}$. The motor rotation speed is controlled by a generator. The cavity rotation speed is such that the sphere is centrifuged and rotates without touching the side walls. The sphere angular velocity $f_{s}=\Omega_{s} / 2 \pi$ is different from the cavity rotation speed. The stroboscopic light lamp 5 is used for illumination. The electrodynamic vibrator produces the translational vibrations of a given frequency $f_{\mathrm{vib}}=\Omega_{\mathrm{vib}} / 2 \pi$. The amplitude of vibration $b_{\mathrm{vib}}$ is calculated using the signal of accelerometer 6 . The cavity inclination angle is measured using a cathetometer accurate to $0.1^{\circ}$.

In the experiment the body position, $x=\left(x_{2}-x_{1}\right) /\left(x_{2}+\right.$ $\left.x_{1}\right)$ with respect to the cavity end walls $\left(x_{1}, x_{2}\right.$; the distance between the left and the right end walls of the cavity to the closest poles of the sphere), and the relative angular velocity of the sphere $\Delta f=f_{s}-f_{\text {rot }}$ is measured. All experiments were conducted in the resonance areas where the frequency of vibrations coincides with one of the natural frequencies of the sphere and the differential rotation $\Delta f$ (outstripping or lagging) being large [5]. For chosen system, the resonant frequencies of outstripping and lagging rotation are $n=2.0$ and $n=0.8$, respectively.

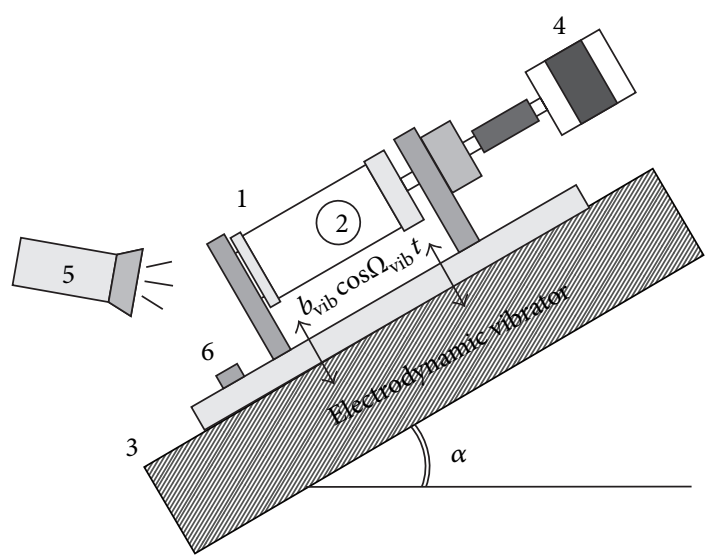

FIGURE 1: The scheme of the experimental setup.

The experiment was conducted as follows. In one case, the angle of the cavity inclination $\alpha$ is changing continuously step by step at constant frequency of rotation and frequency and amplitude of vibrations. The experiment is repeated for different values of $f_{\mathrm{vib}}$ and $b_{\mathrm{vib}}$. In another case, the amplitude of vibration $b_{\text {vib }}$ is varied at definite frequency of vibration and angle $\alpha$.

For the outstripping rotation, the cavity speed and the vibration frequency are $f_{\text {rot }}=17.5 \mathrm{rps}$ and $f_{\text {vib }}=35.0 \mathrm{~Hz}$. For the lagging rotation, $f_{\text {rot }}=31.0 \mathrm{rps}$ and $f_{\text {vib }}=25.0 \mathrm{~Hz}$. The amplitude of the vibrations varies in the range $b_{\mathrm{vib}}=$ $0.10-0.80 \mathrm{~mm}$.

\section{Experimental Results}

At the absence of vibrations, the sphere rotation speed in the laboratory frame is always less than the cavity speed $(\Delta f<0)$ in case of rotation about a horizontal axis. The mechanism of the generation of the body lagging rotation relative to the cavity in the two-dimensional case (the cylindrical body in rotating cylindrical cavity) is described in [4]. The sphere takes a quasistationary position at some distance from the cavity end walls. The position depends on the dimensionless frequency $\omega=\Omega_{\text {rot }} r^{2} / \nu$ where $v$ is the kinematic viscosity.

Vibrational effect is significant only in the narrow resonance areas, when the vibration frequency coincides with the fundamental frequency of the sphere inertial oscillations. In this case, the intensive body differential rotation is excited, which is outstripping at the vibration frequency higher than the rotation frequency $\left(f_{\text {vib }}>f_{\text {rot }}\right)$, or lagging at the reverse ratio of frequencies $\left(f_{\text {vib }}<f_{\text {rot }}\right)$. In the resonance areas, the sphere performs the intensive circularly polarized vibrations with the vibration frequency. The sphere dynamics under vibration depends on the parameter $n$. The area of intense outstripping rotation $(\Delta f>0)$ is characterized by a significant shift of the sphere out of the cavity center (the horizontal case) [5]. The displacement can occur towards any end wall. In the lagging resonant rotation area, the sphere position is at almost equal distance from the ends, $|x| \leq 0.2$. 


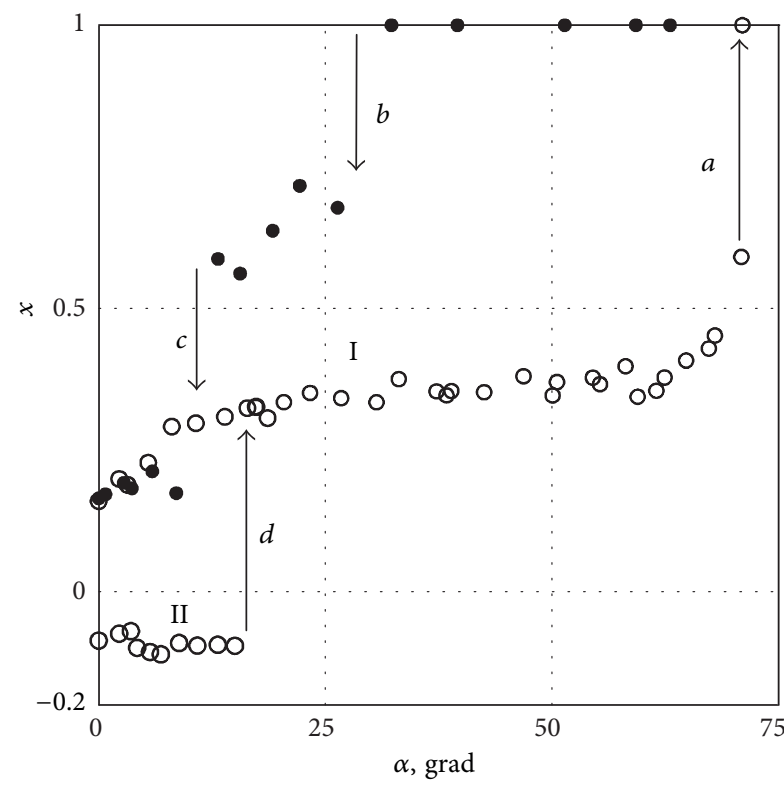

FIGURE 2: The position of the sphere relative to the cavity ends as a function of the angle $\alpha$ for $b_{\text {vib }}=0.50 \mathrm{~mm}$ at $n=0.8$; hereinafter, the empty and dark symbols correspond to the results with increasing and decreasing $\alpha$; the transitions from one quasisteady state to another are shown by arrows.

Outside the resonance areas, the sphere position coincides with the nonvibration case.

For the small amplitudes of vibrations, the sphere retains practically the same quasistationary position with the cavity deviation from the horizon (Figure 2, I). With increasing the cavity inclination, the situation remains unchanged until a certain critical angle $\alpha^{*}$, at which the sphere in the threshold manner moves along the axis to the end wall, "floats" (Figure 2, threshold $a$ ). With decrease of the inclination angle, the separation from the upper wall occurs with hysteresis at much lower value of $\alpha$ (Figure 2, threshold $b$ ). The new quasistationary position of the sphere is much closer to the upper end wall. The transition to the initial state $x$ takes place at threshold $c$. The sphere differential velocity changes simultaneously with the position (Figure 3 ). Floating and separation of the sphere from the top wall are accompanied by the threshold change in the $\Delta f$. With increasing of the range of inclination $\alpha$ the behavior of the sphere becomes unsteady. The rotation speed $\Delta f$ (at $\alpha=$ const) is changing with time while the $x$ remains constant.

In the horizontal cavity, the sphere always occupies a position shifted a little to the left or to the right from the center with equal probability. The experimental points for the symmetric position (Figure 2, II) show that, despite the significant asymmetry, with increasing $\alpha$ the sphere remains unchanged steady-state position closer to the lower end. The transition to a position near the top end (Figure 2, I) is at the threshold $d$.

The increase of the vibration amplitude leads to the growth of the differential rotation speed and the critical angle $\alpha^{*}$. Under the intensive vibration, the sphere could remain

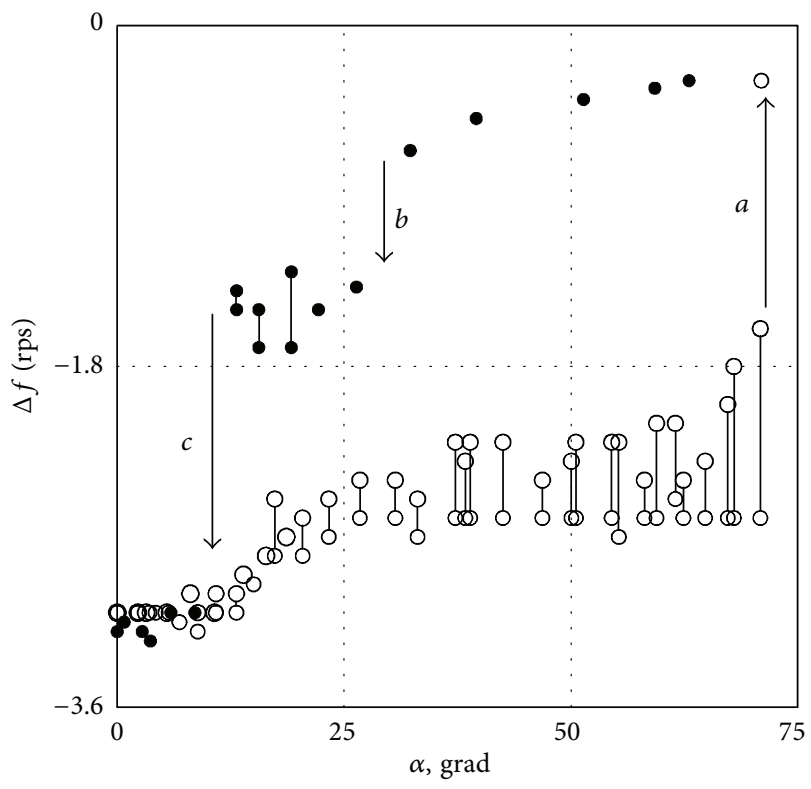

FIGURE 3: The differential rotation speed versus the angle $\alpha$ for $b_{\text {vib }}=$ $0.50 \mathrm{~mm}$ at $n=0.8$; the area of sphere unsteady rotation is indicated by lines connecting the minimum and the maximum values of $\Delta f$.

in the quasiequilibrium position at some distance from the upper end wall of the cavity even in the vertical cylinder (Figure 4(a)). Floating occurs only at low vibration amplitude $b_{\text {vib }}$ (Figure 4(b)). Thus, the vibration allows the suspension of the light sphere in the rotating vertical cylindrical cavity with fluid.

Under vibrations of large amplitude (Figure 5(a)) the sphere is smoothly approaching to the upper end with the increase of the cavity inclination and on reaching the values of $x_{1} \approx 5 \mathrm{~mm}$ is kept at this distance until $\alpha=90^{\circ}$. The relative rotation speed retains the value $\Delta f=-2.3 \mathrm{rps}$ (Figure 5(b)).

\section{Discussion}

The action of the oscillating inertial force, which is associated with the vibrations, on the rotating system depends on the density difference between the body and the fluid, which determines the amplitude of the body oscillations relative to the cavity. If the density of the body coincides with the density of the fluid, then static or oscillating external force fields do not influence the body, which performs the solidstate rotation together with the liquid and the cavity. As shown in [4], the differential rotation speed of the light free body in the case of vibrational hydrodynamic top $\Delta \Omega / \Omega_{\text {rot }} \sim$ $\left(1-\rho_{s} / \rho_{L}\right)^{2}$, where $\rho_{s}$ is the body density and $\rho_{L}$ is the fluid one. The body of low density is free and is near the cavity axis due to the action of the centrifugal force. The problem qualitatively changes if the body is denser than liquid. In this case, the vibrational effects do not occur since the centrifugal force presses the body to the outer cavity boundary.

The action of transversal vibration on the light solid in rotating cavity with liquid is determined by the dimensionless parameter $\Gamma_{\mathrm{vib}}=b_{\mathrm{vib}} \Omega_{\mathrm{vib}}^{2} /\left(r \Omega_{\mathrm{rot}}^{2}\right)$ [5] that characterizes 


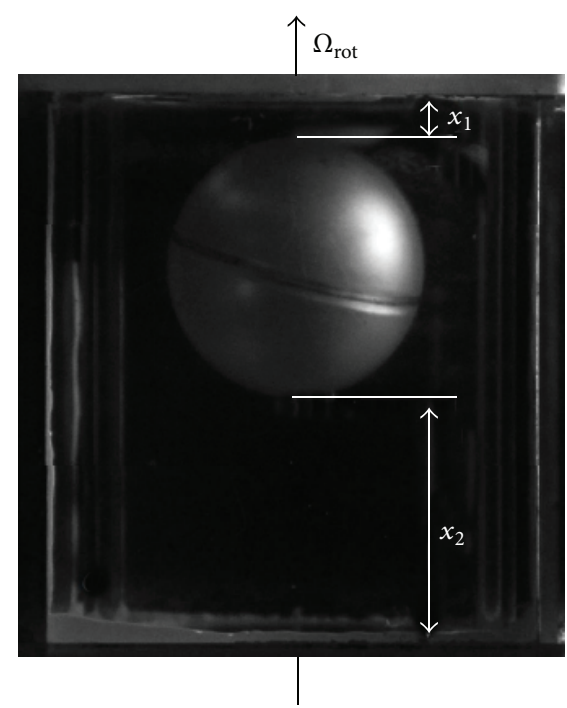

(a)

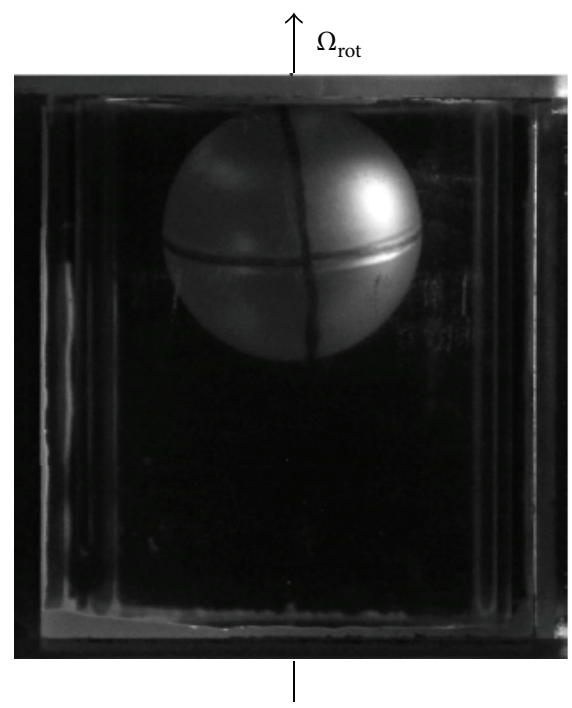

(b)

Figure 4: Photos of the sphere quasistationary position at $n=0.8 ; b_{\mathrm{vib}}=0.82$ (a) and $0.66 \mathrm{~mm}(\mathrm{~b})$ in the cavity rotating around the vertical axis.

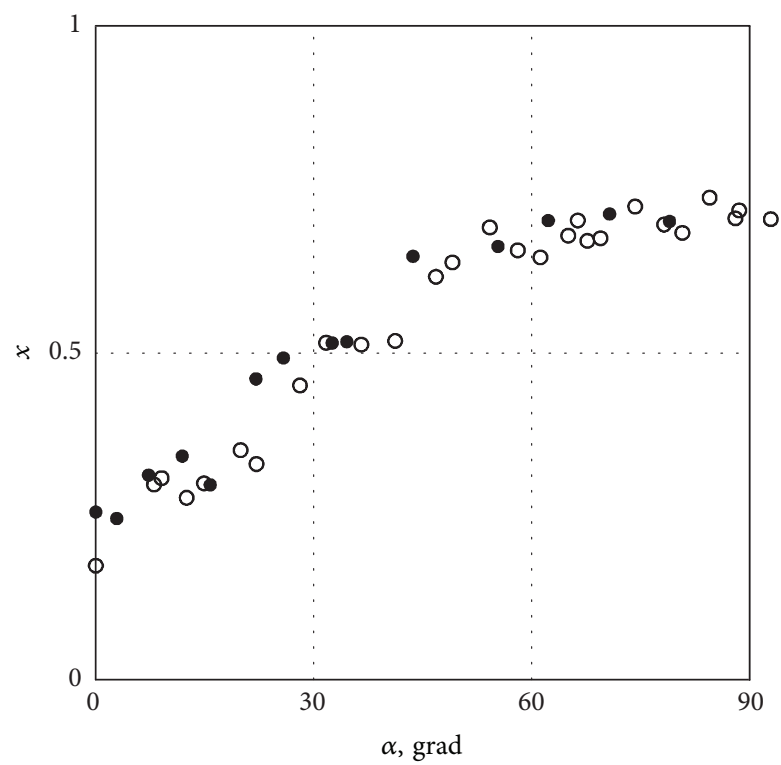

(a)

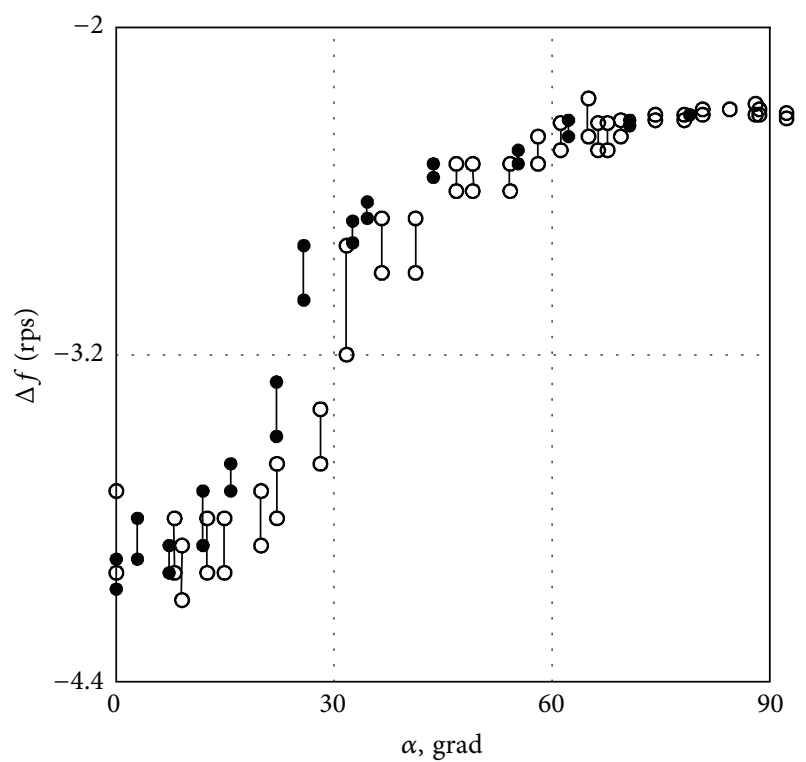

(b)

Figure 5: The position of the sphere relative to the cavity ends (a) and the differential rotation speed (b) as function of the angle $\alpha$ for $b_{\text {vib }}=0.70 \mathrm{~mm}$ at $n=0.8$.

the ratio of the vibrational acceleration to the centrifugal one. The increase of $\Gamma_{\text {vib }}$ leads to the growth of dimensionless velocity of the differential rotation $\left|\Delta \Omega / \Omega_{\text {rot }}\right|$. In the inclined cavity, it also leads to a change in the sphere position $x$. The position $x$ in the horizontal cavity at $n=0.8$ practically does not depend on $\Gamma_{\text {vib }}$.

For different inclination angels, the coordinate $x$ changes with $\Delta \Omega / \Omega_{\text {rot }}$ nonmonotonously. In the case of large angles $\left(\alpha>50^{\circ}\right)$ all data points in complete agreement with each other form a dependence (Figure 6, I). In this case, axial component of the lift force $F_{n}=\left(\rho_{L}-\rho_{S}\right) V g \sin \alpha$, increasing with increasing $\alpha$, is irrelevant. With the decrease in the intensity of the differential rotation, the sphere moves to the upper end of the cavity. Complete transition occurs in a threshold manner.

A specific feature of small angles $\left(\alpha<20^{\circ}\right)$ is the existence of several areas of quasistationary positions of the sphere (Figure 6, II-IV). So reduction of $\left|\Delta \Omega / \Omega_{\text {rot }}\right|$ leads to displacement of the sphere from the upper end to the center of the cavity (curve II). However, at the threshold 


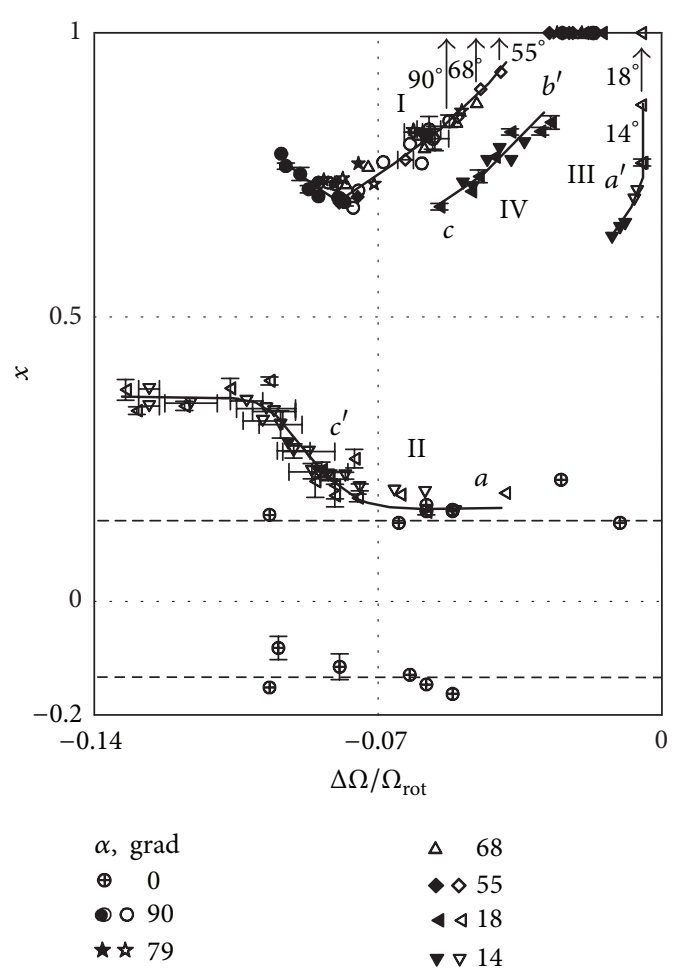

FIGURE 6: The position of the body versus the dimensionless sphere speed at different $\alpha(n=0.8)$; dark and light symbols correspond to increase and decrease of $\Gamma_{\mathrm{vib}}$; solid arrows indicate the thresholds of the sphere float to the upper end wall.

$a$ the sphere from the curve II moves to the point $a^{\prime}$ on the curve III. The new position is much closer to the upper end. With increasing $\left|\Delta \Omega / \Omega_{\text {rot }}\right|$ return to the curve II comes after a series of transitions $b-b^{\prime}$ and $c-c^{\prime}$.

The moderate angles on the plane $x\left(\Delta \Omega / \Omega_{\text {rot }}\right)$ are intermediate between the large and small $\alpha$ (Figure 7). In the area $x>0.5$ the sphere shifts to the upper end wall with increase of the vibrations intensity; at that the relative speed decreases. In this case, the sphere behaves like it does at large $\alpha$. The decrease of the vibration intensity changes the dependency $x\left(\Delta \Omega / \Omega_{\text {rot }}\right)$ to the opposite. The sphere gradually shifts to the cavity center and the relative rotation speed decreases. In the area $x<0.5$ the sphere behaves the same as at small inclination angle.

The position of the body in the cavity is determined by the interaction with the cavity walls, that is, by the structure of averaged flows. The mechanism of the flows generation is associated with the occurrence of the viscous stresses on the body surface as a result of its oscillation relative to the cavity [4]. The averaged viscous stresses result in rotation of both the body and the fluid. Thus, the intensity of the flow is determined by the dimensionless body rotation speed $\Delta \Omega / \Omega_{\text {rot }}$.

In [11], it is shown that at relatively weak differential rotation of the body $\left|\Delta \Omega / \Omega_{\text {rot }}\right| \sim 10^{-2}$ and moderate dimensionless frequencies $\omega \sim 10^{3}-10^{4}$ the averaged flow has the shape of a Taylor-Proudman column. The column

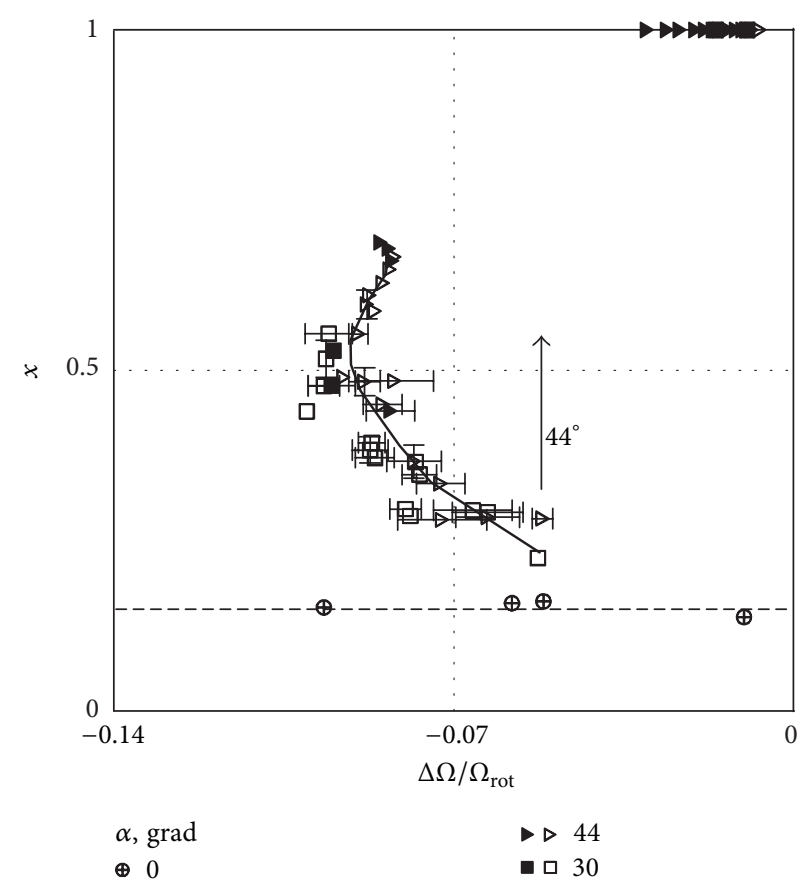

FIgURE 7: The position of the body versus the dimensionless sphere speed for moderate angles; dark and light symbols correspond to the increase and decrease of $\Gamma_{\text {vib }}$.

is elongated along the rotation axis; its transverse size is determined by the body diameter. On the lateral column boundary, the the discontinuity of tangential component of the velocity takes place. Inside the column the averaged flows exist that provides the transfer of momentum, and also the specific vortex flows exist. These flows keep the sphere in the certain quasistationary position relative to the cavity ends. The asymmetric position is associated with the asymmetry of the flows. As shown in [11] at $\left|\Delta \Omega / \Omega_{\text {rot }}\right|>10^{-1}$ and high dimensionless frequencies, $\omega>10^{4}$, the TaylorProudman column is destroyed, and the two-dimensional flow is replaced by an intense three-dimensional circulation. We can assume that these vortex flows are responsible for the forces acting on the body and determine the "suspended" state of the body in the vertical position of the cylinder. In this case, the transitions between different quasistationary positions are associated with qualitative change of the flows structure. This is confirmed by the discrete nature of the transitions between the curves II-IV (Figure 6). The study of the flows structure in the inclined cylinder is a subject of further research.

\section{Conclusions}

The behavior of the low density spherical body in the rotating inclined cylinder filled with fluid under transversal vibration is experimentally investigated. It is found that the vibration results in the intensive differential rotation of the sphere and quasistationary positioning at definite distance from the end walls. With increase of the cavity inclination angle, the sphere 
remains in a suspended state until some critical value $\alpha^{*}$, which grows with the vibration intensity. At high vibration intensity the suspension is possible of the light sphere even when the cavity rotates around the vertical axis.

\section{Conflict of Interests}

The authors declare that there is no conflict of interests regarding the publication of this paper.

\section{Acknowledgments}

The work was done in the frame of Strategic Development Program of PSHPU (Project no. 030-F) with partial support of Ministry of Education of Perm Region (Project no. C26/625) and RFBR (Grant no. 12-01-31521).

\section{References}

[1] I. I. Blekhman, Vibrational Mechanics: Nonlinear Dynamics Effects, General Approach, Applications, World Scientific, Singapore, 2000.

[2] A. A. Ivanova, V. G. Kozlov, and D. A. Polezhaev, "Vibrational dynamics of a centrifuged fluid layer," Fluid Dynamics, vol. 40, no. 2, pp. 297-304, 2005.

[3] A. Salnikova, N. Kozlov, A. Ivanova, and M. Stambouli, "Dynamics of rotating two-phase system under transversal vibration," Microgravity Science and Technology, vol. 21, no. 1-2, pp. 83-87, 2009.

[4] V. G. Kozlov and N. V. Kozlov, "Vibrational hydrodynamic gyroscope," Doklady Physics, vol. 52, no. 8, pp. 458-461, 2007.

[5] A. A. Ivanova, N. V. Kozlov, and S. V. Subbotin, "Vibrational dynamics of a light spherical body in a rotating cylinder filled with a fluid," Fluid Dynamics, vol. 47, no. 6, pp. 683-693, 2012.

[6] E. H. Trinh and C. J. Hsu, "Equilibrium shapes of acoustically levitated drops," Journal of the Acoustical Society of America, vol. 79, no. 5, pp. 1335-1338, 1986.

[7] E. H. Trinh and T. G. Wang, "Large-amplitude free and driven drop-shape oscillations: experimental observations," Journal of Fluid Mechanics, vol. 122, pp. 315-338, 1982.

[8] G. I. Taylor, "The motion of a sphere in a rotating liquid," Proceedings of the Royal Society A, vol. 102, no. 715, pp. 180-189, 1922.

[9] T. Maxworthy, "The observed motion of a sphere through a short, rotating cylinder of fluid," Journal of Fluid Mechanics, vol. 31, no. 4, pp. 643-655, 1968.

[10] H. P. Greenspan, The Theory of Rotating Fluids, Cambridge University Press, London, UK, 1968.

[11] V. G. Kozlov, N. V. Kozlov, and S. V. Subbotin, "Motion of fluid and a solid core in a spherical cavity rotating in an external force field," Doklady Physics, vol. 59, no. 1, pp. 40-44, 2014. 

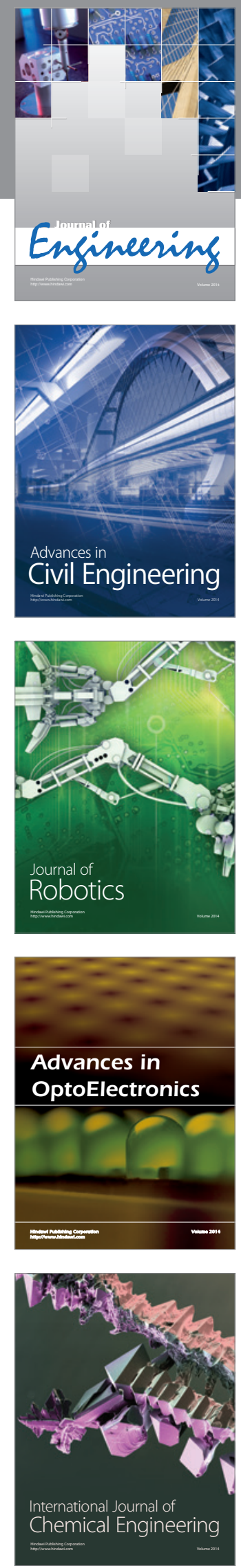

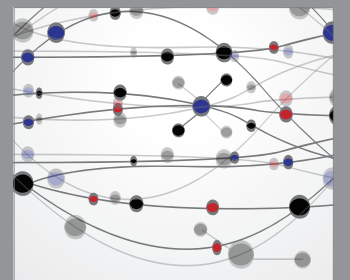

The Scientific World Journal
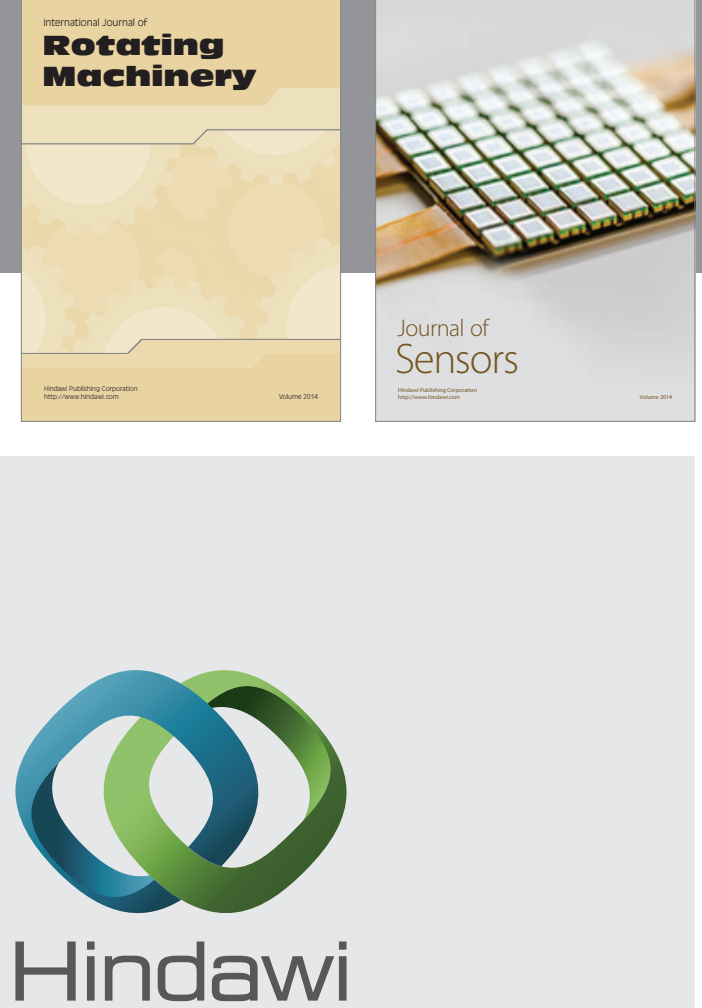

Submit your manuscripts at http://www.hindawi.com
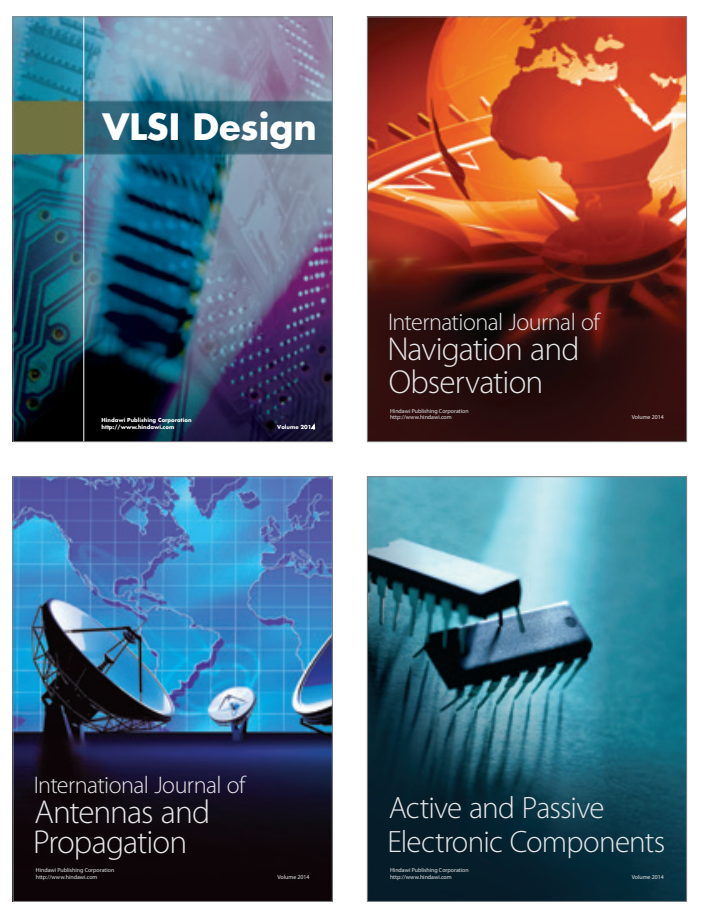
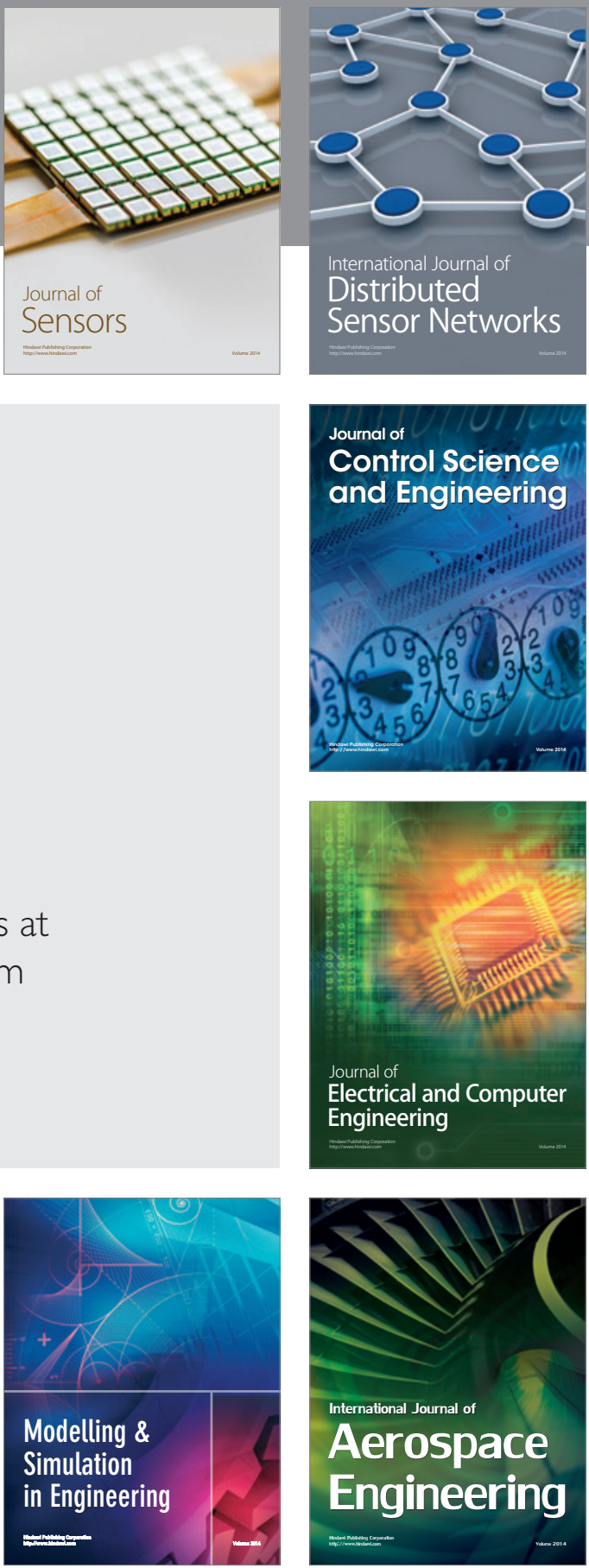

Journal of

Control Science

and Engineering
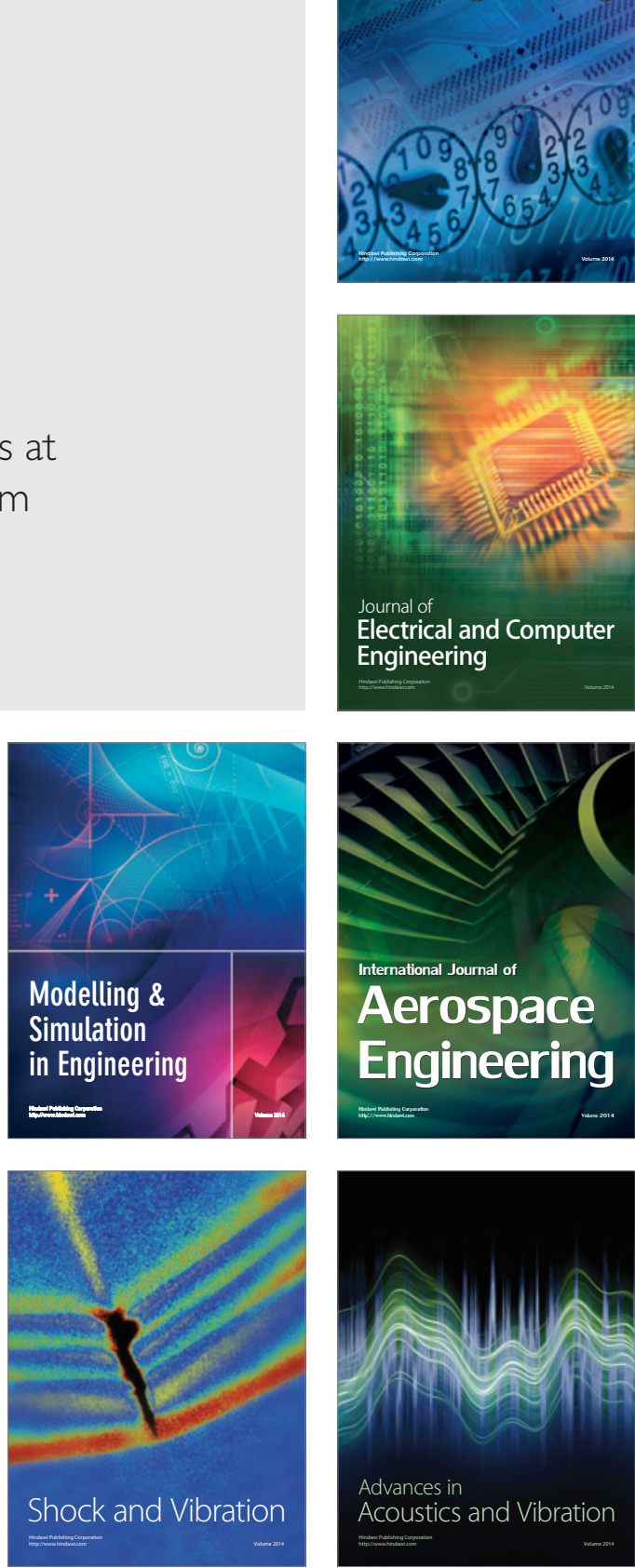